\title{
RADIAL HEAT CONDUCTION IN A MULTILAYERED SPHERE
}

\author{
Urszula Siedlecka \\ Institute of Mathematics, Czestochowa University of Technology \\ Częstochowa, Poland \\ urszula.siedlecka@im.pcz.pl
}

\begin{abstract}
In this paper, an exact analytical solution of the radial heat conduction problem in a hollow multilayered sphere is presented. The boundary conditions of the third kind and perfect contact at the interfaces are assumed. The temperatures are obtained by using the Green's function method. The Green's functions are expressed in the form of the series of appropriate eigenfunctions.
\end{abstract}

Keywords: Green's function, heat conduction, multilayered hollow sphere

\section{Introduction}

Thermal analysis of composite media is of great importance since it has been widely used in real physical and engineering systems. The knowledge of the temperature distribution in the composite material is a basis to the thermal analysis. The heat conduction in composite slabs, cylinders and spheres were the subject of consideration by much research. In particular, the authors of the references [1-5] deal with the heat conduction in hollow and solid spheres.

The heat conduction in a sphere can be considered in one-, two- or three-dimensions. A solution to one-dimensional, radial heat conduction problem of a solid sphere with internal heat generation was presented by Pawar et al. in the paper [1]. An integral transform technique was applied to obtain the temperature distribution in the sphere. An analytical method based on separation of variables and finite integral transform has been used to obtain the solution of the heat conduction in the hollow sphere in the reference [2]. The heat conduction in layered spheres with time-dependent boundary conditions was considered by Lu and Viljanen in paper [3]. The authors use an analytical method to derive a closed form approximate solution of the radial heat conduction problem. In the book [4] by Beck et al. the Green's function method is used to solve the heat conduction problems. This method has been applied in the book [5] by Özişik to determine the temperature distribution in the layered slabs, cylinders and spheres with internal heat generation.

The aim of this paper is to develop an exact analytical solution of the radial heat conduction problem in a hollow multilayered sphere. The solution has been obtained by using the Green's function method. 


\section{Formulation of the problem}

Consider a $N$-layered hollow sphere, a schematic diagram of which is shown in Figure 1. The governing equation to radial heat conduction in spherical coordinates is [4]:

$$
\frac{1}{r^{2}} \frac{\partial}{\partial r}\left(r^{2} \frac{\partial T_{i}}{\partial r}\right)+\frac{1}{\lambda_{i}} g_{i}(r, t)=\frac{1}{\alpha_{i}} \frac{\partial T_{i}}{\partial t}, \quad r \in\left[r_{i-1}, r_{i}\right], \quad i=1, \ldots, N
$$

where $\alpha_{i}$ and $\lambda_{i}$ are the thermal diffusivity and thermal conductivity, respectively, $r$ is the spherical coordinate, $\delta(\cdot)$ is the Dirac delta function, $g_{i}$ is the heat generation, $T_{i}$ is the temperature in the $i$-th layer. The equation (1) can be rewritten in the form

$$
\frac{1}{r} \frac{\partial^{2}}{\partial r^{2}}\left(r T_{i}\right)+\frac{1}{\lambda_{i}} g_{i}(r, t)=\frac{1}{\alpha_{i}} \frac{\partial T_{i}}{\partial t}, \quad r \in\left[r_{i-1}, r_{i}\right], \quad i=1, \ldots, N
$$

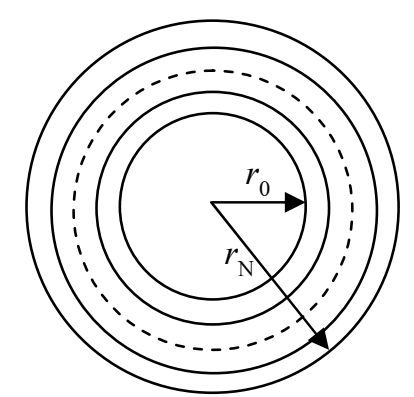

Fig. 1. A schematic diagram of the $N$-layered hollow sphere

In this paper, we assume convective conditions at the inner and outer surfaces of the hollow sphere, a perfect contact at the interfaces of the layers and the initial condition given in each layer:

$$
\begin{gathered}
\lambda_{1} \frac{\partial T_{1}}{\partial r}\left(r_{0}, t\right)=-\alpha_{+}\left(T_{+}(t)-T_{1}\left(r_{0}, t\right)\right) \\
-\lambda_{N} \frac{\partial T_{N}}{\partial r}\left(r_{N}, t\right)=-\alpha_{\infty}\left(T_{\infty}(t)-T_{N}\left(r_{N}, t\right)\right), \\
T_{i}\left(r_{i}, t\right)=T_{i+1}\left(r_{i}, t\right), \quad i=1, \ldots, N-1 \\
\lambda_{i} \frac{\partial T_{i}}{\partial r}\left(r_{i}, t\right)=\lambda_{i+1} \frac{\partial T_{i+1}}{\partial r}\left(r_{i}, t\right), \quad i=1, \ldots, N-1 \\
T_{i}(r, 0)=F_{i}(r), \quad r \in\left[r_{i-1}, r_{i}\right], \quad i=1, \ldots, N
\end{gathered}
$$


where $\alpha_{+}, \alpha_{\infty}$ are the heat transfer coefficients, $T_{+}$and $T_{\infty}$ are the inner and outer boundary temperatures, respectively, $F_{i}(r)$ is the initial temperature in the $i$-th layer.

Introducing the functions

$$
V_{i}(r, t)=r T_{i}(r, t), \quad r \in\left[r_{i-1}, r_{i}\right], \quad i=1, \ldots, N
$$

into equations (1)-(7), we obtain the initial - boundary problem with the constant coefficients differential equation for the function $V_{i}$ :

$$
\begin{gathered}
\frac{\partial^{2} V_{i}}{\partial r^{2}}+\frac{r}{\lambda_{i}} g_{i}(r, t)=\frac{1}{\alpha_{i}} \frac{\partial V_{i}}{\partial t}, \quad r \in\left[r_{i-1}, r_{i}\right], \quad i=1, \ldots, N \\
\frac{\partial V_{1}\left(r_{0}, t\right)}{\partial r}=\left(\frac{1}{r_{0}}+\frac{\alpha_{+}}{\lambda_{1}}\right) V_{1}\left(r_{0}, t\right)-\frac{\alpha_{+}}{\lambda_{1}} r_{0} T_{+}(t) \\
V_{i}\left(r_{i}, t\right)=V_{i+1}\left(r_{i}, t\right), \quad i=1, \ldots, N-1 \\
\frac{\lambda_{i}}{\lambda_{i+1}} \frac{\partial V_{i}\left(r_{i}, t\right)}{\partial r}+r_{i}^{-1}\left(1-\frac{\lambda_{i}}{\lambda_{i+1}}\right) V_{i}\left(r_{i}, t\right)=\frac{\partial V_{i+1}\left(r_{i}, t\right)}{\partial r}, \quad i=1,2, \ldots, N-1 \\
\frac{\partial V_{N}\left(r_{N}, t\right)}{\partial r}=\left(\frac{1}{r_{N}}-\frac{\alpha_{\infty}}{\lambda_{N}}\right) V_{N}\left(r_{N}, t\right)+\frac{\alpha_{\infty}}{\lambda_{N}} r_{N} T_{\infty}(t) \\
V_{i}(r, 0)=r F_{i}(r), \quad r \in\left[r_{i-1}, r_{i}\right], \quad i=1, \ldots, N
\end{gathered}
$$

To solve the problem (9)-(14), we use the Green's function method. For this purpose, we consider an auxiliary problem to determine the Green's functions.

\section{Derivation of the Green's functions}

The Green's functions $G_{i, j}\left(r, t ; r^{\prime}, \tau\right)$ associated with the problem (9)-(14) satisfy the differential equation

$$
\frac{\partial^{2} G_{i, j}}{\partial r^{2}}+\frac{1}{\alpha_{i}} \delta\left(r-r^{\prime}\right) \delta(t-\tau)=\frac{1}{\alpha_{i}} \frac{\partial G_{i, j}}{\partial t}, \quad r \in\left[r_{i-1}, r_{i}\right], \quad i=1, \ldots, N
$$

and the zero initial condition and the following homogeneous boundary conditions 


$$
\begin{gathered}
\frac{\partial G_{1, j}\left(r_{0}, t ; r^{\prime}, \tau\right)}{\partial r}=\left(\frac{1}{r_{0}}+\frac{\alpha_{+}}{\lambda_{1}}\right) G_{1, j}\left(r_{0}, t ; r^{\prime}, \tau\right) \\
G_{i, j}\left(r_{i}, t ; r^{\prime}, \tau\right)=G_{i+1, j}\left(r_{i}, t ; r^{\prime}, \tau\right), \quad r^{\prime} \in\left[r_{j-1}, r_{j}\right], \quad i=1, \ldots, N-1, \quad j=1, \ldots, N \\
\frac{\lambda_{i}}{\lambda_{i+1}} \frac{\partial G_{i, j}\left(r_{i}, t ; r^{\prime}, \tau\right)}{\partial r}+r_{i}^{-1}\left(1-\frac{\lambda_{i}}{\lambda_{i+1}}\right) G_{i, j}\left(r_{i}, t ; r^{\prime}, \tau\right)=\frac{\partial G_{i+1, j}\left(r_{i}, t ; r^{\prime}, \tau\right)}{\partial r} \\
\frac{\partial G_{N, j}\left(r_{N}, t ; r^{\prime}, \tau\right)}{\partial r}=\left(\frac{1}{r_{N}}-\frac{\alpha_{\infty}}{\lambda_{N}}\right) G_{N, j}\left(r_{N}, t ; r^{\prime}, \tau\right)
\end{gathered}
$$

To determine the Green's functions $G_{i, j}$, first, we solve the following eigenproblem:

$$
\begin{gathered}
\frac{d^{2} \Phi_{i}(r)}{d r^{2}}+\frac{\beta^{2}}{\alpha_{i}} \Phi_{i}(r)=0 \quad r \in\left[r_{i-1}, r_{i}\right], \quad i=1, \ldots, N \\
\frac{d \Phi_{1}\left(r_{0}\right)}{d r}=\left(\frac{1}{r_{0}}+\frac{\alpha_{+}}{\lambda_{1}}\right) \Phi_{1}\left(r_{0}\right) \\
\Phi_{i}\left(r_{i}\right)=\Phi_{i+1}\left(r_{i}\right), \quad i=1, \ldots, N-1+ \\
\lambda_{i} \frac{d \Phi_{i}\left(r_{i}\right)}{d r}+r_{i}^{-1}\left(\lambda_{i+1}-\lambda_{i}\right) \Phi_{i}\left(r_{i}\right)=\lambda_{i+1} \frac{d \Phi_{i+1}\left(r_{i}\right)}{d r}, \quad i=1,2, \ldots, N-1 \\
\frac{d \Phi_{N}\left(r_{N}\right)}{d r}=\left(\frac{1}{r_{N}}-\frac{\alpha_{\infty}}{\lambda_{N}}\right) \Phi_{N}\left(r_{N}\right)
\end{gathered}
$$

where $\beta$ is a constant.

The general solution of differential equation (20) has the form

$$
\Phi_{i}(r)=A_{i} \cos \mu_{i} r+B_{i} \sin \mu_{i} r, \quad r \in\left[r_{i-1}, r_{i}\right], \quad i=1, \ldots, N
$$

where $\mu_{i}=\frac{\beta}{\sqrt{\alpha_{i}}}$. Substituting the functions $\Phi_{i}$ into boundary conditions (21)-(24), we obtain a system of equations with respect to constants $A_{i}, B_{i}(i=1, \ldots, N)$ : 


$$
\begin{gathered}
{\left[\lambda_{1} \mu_{1} \sin \mu_{1} r_{0}+\left(\frac{1}{r_{0}}+\frac{\alpha_{+}}{\lambda_{1}}\right) \cos \mu_{1} r_{0}\right] A_{1}-} \\
-\left[\lambda_{1} \mu_{1} \cos \mu_{1} r_{0}-\left(\frac{1}{r_{0}}+\frac{\alpha_{+}}{\lambda_{1}}\right) \sin \mu_{1} r_{0}\right] B_{1}=0 \\
A_{i} \cos \mu_{i} r_{i}+B_{i} \sin \mu_{i} r_{i}-A_{i+1} \cos \mu_{i+1} r_{i}-B_{i+1} \sin \mu_{i+1} r_{i}=0 \\
\quad i=1, \ldots, N-1 \\
-\lambda_{i}\left(\mu_{i} \sin \mu_{i} r_{i}+r_{i}^{-1} \cos \mu_{i} r_{i}\right) A_{i}+\lambda_{i}\left(\mu_{i} \cos \mu_{i} r_{i}-r_{i}^{-1} \sin \mu_{i} r_{i}\right) B_{i} \\
+\lambda_{i+1}\left(\mu_{i+1} \sin \mu_{i+1} r_{i i}+r_{i}^{-1} \cos \mu_{i+1} r_{i}\right) A_{i+1} \\
-\lambda_{i+1}\left(\mu_{i+1} \cos \mu_{i+1} r_{i}-r_{i}^{-1} \sin \mu_{i+1} r_{i}\right) B_{i+1}=0 \\
{\left[\mu_{N} \sin \mu_{N} r_{N}+\left(\frac{1}{r_{N}}-\frac{\alpha_{\infty}}{\lambda_{N}}\right) \cos \mu_{N} r_{N}\right] A_{N}} \\
-\left[\mu_{N} \cos \mu_{N} r_{N}-\left(\frac{1}{r_{N}}-\frac{\alpha_{\infty}}{\lambda_{N}}\right) \sin \mu_{N} r_{N}\right] B_{N}=0
\end{gathered}
$$

The equation system (26)-(29) can be written in the matrix form

$$
\mathbf{A C}=\mathbf{0}
$$

where $\mathbf{C}=\left[\begin{array}{llllll}A_{1} & B_{1} & A_{2} & B_{2} \ldots & A_{N} & B_{N}\end{array}\right]^{T}$ and the non-zero elements $a_{i j}, i, j=1, \ldots, 2 N$ of the matrix $\mathbf{A}$ are the coefficient at the constants $A_{i}, B_{i}$. The non-zero solution of the equation (30) exists for these values $\beta$, for which the determinant of the matrix $\mathbf{A}$ vanishes, i.e.

$$
\operatorname{det} \mathbf{A}=0
$$

The equation (31) is then solved numerically with respect to $\beta$. For each eigenvalue $\beta_{k}$, the corresponding eigenfunction $\Phi(r)=\Phi_{i}(r)$ for $r \in\left[r_{i-1}, r_{i}\right], i=1,2, \ldots, N$, where $\Phi_{i}$ are given by equation (25). The coefficients $A_{i}, B_{i}$ occurring in the equation (25) for each $\beta_{k}$ are determined by solving an equation system which is obtained by assuming $\beta=\beta_{k}, B_{N}=1$ and omitting the last equation in the system (30). 
The functions $\Phi_{i, k}$ satisfy the orthogonality condition:

$$
\sum_{i=1}^{N} \frac{\lambda_{i}}{\alpha_{i}} \int_{r_{i-1}}^{r_{i}} \Phi_{i, k}(r) \Phi_{i, k^{\prime}}(r) d r=\left\{\begin{array}{lll}
0 & \text { for } & k^{\prime} \neq k \\
N_{k} & \text { for } & k^{\prime}=k
\end{array}\right.
$$

where

$$
\begin{aligned}
N_{k}=\frac{1}{4 \mu_{i, k}} & {\left[2\left(A_{i, k}^{2}+B_{i, k}^{2}\right)\left(r_{i}-r_{i-1}\right) \mu_{i}-2 A_{i, k} B_{i, k}\left(\cos 2 r_{i} \mu_{i, k}-\cos 2 r_{i-1} \mu_{i, k}\right)\right.} \\
& \left.+\left(A_{i, k}^{2}-B_{i, k}^{2}\right)\left(\sin 2 r_{i} \mu_{i, k}-\sin 2 r_{i-1} \mu_{i, k}\right)\right]
\end{aligned}
$$

The Green's functions $G_{i, j}$ can be expressed in the form of the series of eigenfunctions $\Phi_{i, k}(r)$ :

$$
\begin{gathered}
G_{i, j}\left(r, t ; r^{\prime}, \tau\right)=\sum_{k=1}^{\infty} \Gamma_{k}(t, \tau) \Phi_{i, k}(r) \Phi_{j, k}\left(r^{\prime}\right), \\
\quad r \in\left[r_{i-1}, r_{i}\right], \quad r^{\prime} \in\left[r_{j-1}, r_{j}\right], \quad i, j=1, \ldots, N
\end{gathered}
$$

Substituting the series (33) into equation (15) and using the orthogonality condition (32), we obtain an equation for the functions $\Gamma_{k}(t, \tau)$ in the form

$$
\frac{\partial \Gamma_{k}(t, \tau)}{\partial t}+\beta_{k}^{2} \Gamma_{k}(t, \tau)=\frac{1}{N_{k}} \delta(t-\tau)
$$

The solution of this equation is

$$
\Gamma_{k}(t, \tau)=\frac{1}{N_{k}} \exp \left(-\beta_{k}^{2}(t-\tau)\right)
$$

Finally, the Green's functions for the heat conduction problems in the multilayer hollow sphere with boundary conditions of the third kind and perfect contact at the interfaces have the form

$$
\begin{array}{r}
G_{i, j}\left(r, t ; r^{\prime}, \tau\right)=\frac{\lambda_{j}}{\alpha_{j}} \sum_{k=1}^{\infty} \frac{1}{N_{k}} \exp \left(-\beta_{k}^{2}(t-\tau)\right) \Phi_{i, k}(r) \Phi_{j, k}\left(r^{\prime}\right), \\
r \in\left[r_{i-1}, r_{i}\right], \quad r^{\prime} \in\left[r_{j-1}, r_{j}\right], \quad i, j=1, \ldots, N
\end{array}
$$

Using the Green's functions (36), the solution of the initial-boundary problem (9)-(14) can be presented in the form 


$$
\begin{aligned}
& V_{i}(r, t)=\frac{\alpha_{i}}{\lambda_{i}} \sum_{j=1}^{N} \lambda_{j} \int_{0}^{t}\left[\left.\frac{\partial V_{i}\left(r^{\prime}, \tau\right)}{\partial r^{\prime}} G_{i, j}\left(r_{i}, t ; r^{\prime}, \tau\right)\right|_{r^{\prime}=r_{j-1}} ^{r_{j}}-\left.\frac{\partial G_{i, j}}{\partial r^{\prime}} V_{j}\left(r^{\prime}, \tau\right)\right|_{r^{\prime}=r_{j-1}} ^{r^{\prime}=r}\right] d \tau \\
& +\sum_{j=1}^{N} \frac{\alpha_{j}}{\lambda_{j}} \int_{0}^{t} \int_{r_{j-1}}^{r_{j}} r^{\prime} g_{i}\left(r^{\prime}, \tau\right) G_{i, j}\left(r_{i}, t ; r^{\prime}, \tau\right) d r^{\prime} d \tau+ \\
& -\left.\sum_{j=1}^{N} \int_{r_{j-1}}^{r_{j}} V_{i}\left(r^{\prime}, \tau\right) G_{i, j}\left(r_{i}, t ; r^{\prime}, \tau\right)\right|_{\tau=0} ^{\tau=t} d r^{\prime}
\end{aligned}
$$

As a result, the temperature $T_{i}(r, t)$ in the $i$-th layer obtained on the basis of equations ( 8 ) and (37) is given by:

$$
\begin{aligned}
& T_{i}(r, t)=\alpha_{+} \frac{\alpha_{1}}{\lambda_{1}} \frac{r_{0}}{r} \int_{0}^{t} T_{+}(\tau) G_{i, 1}\left(r, t ; r_{0}, \tau\right) d \tau+ \\
& +\alpha_{\infty} \frac{\alpha_{N}}{\lambda_{N}} \frac{r_{N}}{r} \int_{0}^{t} T_{\infty}(\tau) G_{i, N}\left(r, t ; r_{N}, \tau\right) d \tau+ \\
& +\frac{1}{r} \sum_{j=1}^{N} \frac{\alpha_{j}}{\lambda_{j}} \int_{0}^{t} \int_{r_{j-1}}^{r_{j}} r^{\prime} g_{i}\left(r^{\prime}, \tau\right) G_{i, j}\left(r, t ; r^{\prime}, \tau\right) d r^{\prime} d \tau+ \\
& +\frac{1}{r} \sum_{j=1}^{N} \int_{r_{j-1}}^{r_{j}} r^{\prime} F_{i}\left(r^{\prime}\right) G_{i, j}\left(r, t ; r^{\prime}, 0\right) d r^{\prime}
\end{aligned}
$$

\section{Conclusions}

An exact solution of the problem of the radial heat conduction in a multilayered hollow sphere by using a Green's function method is obtained. The temperature distribution in the sphere as a function of the radial coordinate and time has been presented in the form of a sum of four components. These components depend on the initial temperature, on the inner and outer ambient temperatures and on the heat source. The Green's functions occurring in the solution of the problem are derived.

The solution of the problem of heat conduction in a solid sphere can be obtained by respectively changing of the boundary conditions at the inner surface of the sphere in the presented formulation. The final temperature function can be used to determine the stresses in the layers of the sphere, as well as for the numerical analysis of the heat conduction in the multilayered sphere.

\section{References}

[1] Pawar S.P., Deshmukh K.C., Kedar G.D., Effect of generation on quasi-static thermal stresses in a solid sphere, IOSR Journal of Mathematics 2013, 7, 5, 21-29. 
[2] Singh S., Analytical solution of the time-dependent multilayered heat conduction problems for nuclear applications, Proceedings of the 1st International Nuclear and Renewable Energy Conference 2010, 1-6.

[3] Lu X., Viljanen M., An analytical method to solve heat conduction in layered spheres with time-dependent boundary conditions, Physics Letters A 2006, 351, 274-282.

[4] Beck J.V., Cole K.D., Haji-Sheikh A., Litkouhi B., Heat Conduction Using Green's Functions, Hemisphere, Washington, DC 1992.

[5] Özişik M.N., Heat Condition, second edition, John Wiley \& Sons, Inc., New York 1993. 